\title{
MORE ON THE BORWEIN-DITOR THEOREM
}

\author{
HARRY I. MILLER AND LEILA MILLER-VAN WIEREN
}

Dedicated to Professor Mustafa Kulenović on the occasion of his $60^{\text {th }}$ birthday

\begin{abstract}
In 1978 D. Borwein and S. Z. Ditor published a paper answering a question of P. Erdos. Since then several authors including N. Bingham, P. Komjath, H. Miller, L. Miller-Van Wieren, A. Ostaszewski have generalized and extended their result. In this paper a significant generalization of all previous results is presented.
\end{abstract}

\section{INTRODUCTION}

D. Borwein and S.Z. Ditor [1] have proved the following theorem, answering a question of P. Erdos.

Theorem 1.1. (Borwein, Ditor 1978)

(1) If $A$ is a measurable set in $R$ with $m(A)>0$, and $\left(d_{n}\right)$ is a sequence of reals converging to 0 , then for almost all $x \in A, x+d_{n} \in A$ for infinitely many $n$.

(2) There exists a measurable set $A$ in $R$ with $m(A)>0$, and a (decreasing) sequence $\left(d_{n}\right)$ converging to 0 , such that, for each $x, x+d_{n} \notin A$ for infinitely many $n$.

In [2] $\mathrm{H}$. Miller extended the Borwein-Ditor theorem by using a general function $f: R X R \longrightarrow R$ instead of addition.

In [3] N. Bingham and A. Ostaszewski considered homotopy and its relation to the Borwein-Ditor theorem.

In [5] H. Miller and A. Ostaszewski considered general spaces, group action and shift-compactness and their relations to the Borwein-Ditor theorem.

In [4] H. Miller and L. Miller-Van Wieren extended a result in the last mentioned paper from $R$ to $R^{2}$.

In this paper we prove a double extension of the original Borwein-Ditor theorem.

2000 Mathematics Subject Classification. 40D25, 40G99, 28A12. 


\section{RESUlT}

In [4], it is shown that if $A$ is any nowhere dense subset of $[0,1]$, then there exists a sequence $\left(d_{n}\right)$ converging to zero such that, for each $x, x+d_{n} \notin A$ for infinitely many $n$. We now present a result analogous to the last mentioned theorem for a general $f, f: R x R \longrightarrow R$, in place of addition.

Theorem 2.1. Suppose $f: R x R \longrightarrow R$ is continuous and satisfies:

(1) there exists $e \in R$ such that $f(x, e)=x$ for all $x \in R$,

(2) there exist $\left(x_{1}, y_{1}\right) \in R^{2}, x_{0} \in R, x_{1}>x_{0}, y_{1}>e$, such that the partial derivatives $f_{x}, f_{y}$ exist and are continuous on $T$ the closed rectangle with corners $\left(x_{0}, e\right),\left(x_{0}, y_{1}\right),\left(x_{1}, e\right)$, and $\left(x_{1}, y_{1}\right)$,

(3) there exist $a, b \in R$, with $a, b>0, b>1$ and $a<f_{x}, f_{y}<b$ on $T$.

If $E \subset\left[x_{0}, x_{1}\right]$ is an arbitrary nowhere dense set, then there exists $\left\{e_{n}\right\}_{n=1}^{\infty}$, monotonically converging to e such that $f\left(x, e_{n}\right) \notin E$ infinitely often for each $x \in R$.

Proof. Suppose $n \geq 2$ is arbitrarily fixed. Divide $\left[x_{0}, x_{1}\right]$ into $2^{n}$ adjoining intervals each of length $\frac{x_{1}-x_{0}}{2^{n}}$ and denote them $\left\{I_{n k}\right\}, k=1,2 \ldots 2^{n}$.

Since $E$ is nowhere dense, each $I_{n k}$ contains an open interval $J_{n k}(k=$ $1,2 \ldots, 2^{n}$ ) disjoint from $E$. Let $s_{n}$ denote the minimum length among the intervals $J_{n_{k}}, k=1,2 \ldots 2^{n}$.

Now suppose $x \in\left[x_{0}, x_{1}-\frac{x_{1}-x_{0}}{2^{n}}\right]$ is arbitrary.

Consider the finite sequence $d_{n}, 2 d_{n}, 3 d_{n}, \ldots, m(n) d_{n}$, where $d_{n}=\frac{s_{n}}{2 b}$ and $m(n)$ is the smallest integer such that $m(n) d_{n} a>\frac{x_{1}-x_{0}}{2^{n-1}}$.

Notice $d_{n}<\frac{s_{n}}{2}$ and from our choice of $m(n)$ we have that $(m(n)-1) d_{n} a \leq$ $\frac{x_{1}-x_{0}}{2^{n-1}}$ from which $m(n) d_{n} \leq \frac{x_{1}-x_{0}}{a 2^{n-1}}+d_{n}$.

Now examine the sequence

$$
f(x, e)=x, f\left(x, e+d_{n}\right), f\left(x, e+2 d_{n}\right), \ldots, f\left(x, e+m(n) d_{n}\right) .
$$

Since $x \in I_{n_{k}}$ for some $k \in\left\{1,2, \ldots, 2^{n}-1\right\}$, then we claim that the above sequence is strictly increasing and the difference of successive terms is less than $\frac{s_{n}}{2}$ and $f\left(x, e+m(n) d_{n}\right)-f(x, e)>\frac{2\left(x_{1}-x_{0}\right)}{2^{n}}$.

To see this observe:

$$
\frac{s_{n}}{2}=b d_{n}>f\left(x, e+(k+1) d_{n}\right)-f\left(x, e+k d_{n}\right)>a d_{n}>0
$$

for $0 \leq k \leq m(n)-1$ and

$$
\begin{aligned}
f\left(x, e+m(n) d_{n}\right)-f(x, e) & >m(n) a d_{n}>\frac{x_{1}-x_{0}}{2^{n-1}} \\
& >\frac{2\left(x_{1}-x_{0}\right)}{2^{n}} .
\end{aligned}
$$


So if $x \in\left[x_{0}, x_{1}-\frac{x_{1}-x_{0}}{2^{n}}\right]$, there exists a $j_{x} \in\{0,1, \ldots, m(n)\}$ such that $f\left(x, e+j_{x} d_{n}\right) \notin E$.

The set $\bigcup_{n=1}^{\infty} \bigcup_{k=1}^{m(n)}\left\{k d_{n}\right\}$ can be arranged as a monotonic non-increasing sequence $\left\{h_{n}\right\}_{n=1}^{\infty}$ converging to zero (which is clear from earlier computations) and let $\left\{e_{n}\right\}_{n=1}^{\infty}=\left\{e+h_{n}\right\}_{n=1}^{\infty}$. Then clearly, for each $x \in\left[x_{0}, x_{1}\right)$, $f\left(x, e_{n}\right) \notin E$ for infinitely many $n$. The same is trivially true for $x_{1}$, due to the positive partial derivatives at $\left(x_{1}, e\right)$ and for $x \notin\left[x_{0}, x_{1}\right]$, due to the continuity of $f$.

This completes the proof.

\section{REFERENCES}

[1] D. Borwein and S. Z. Ditor, Translates of sequences in sets of positive measure, Canad. Math. Bull., 21 (1978), 497-498.

[2] H.I. Miller, Generalization of a result of Borwein and Ditor, Proc. Amer. Math. Soc., 105 (4) (1989), 889-893.

[3] N.H. Bingham and A.J. Ostazewski, Homotopy and the Kestelman-Borwein-Ditor theorem, Canad. Math. Bull., 54 (1) (2011), 12-20.

[4] H. I. Miller and L. Miller-Van Wieren, Translates of sequences for some small sets, Sarajevo J. Math., 7 (2) (2011), 201-205.

[5] H.I. Miller and A.J. Ostaszewski, Group action, shift compactness and the KBD theorem, JMAA, online (2012), 22-39.

(Received: July 18, 2012)

Faculty of Engineering and Natural Sciences International University of Sarajevo

Sarajevo, 71000

Bosnia-Herzegovina

E-mails: himiller@hotmail.com lejla.miller@yahoo.com 